\title{
Red Blood Cell Destruction in Autoimmune Hemolytic Anemia: Role of Complement and Potential New Targets for Therapy
}

\author{
Sigbjørn Berentsen ${ }^{1}$ and Tatjana Sundic ${ }^{2}$ \\ ${ }^{1}$ Department of Medicine, Haugesund Hospital, Helse Fonna, P.O. Box 2170, 5504 Haugesund, Norway \\ ${ }^{2}$ Department of Immunology and Transfusion Medicine, Haugesund Hospital, Helse Fonna, P.O. Box 2170, 5504 Haugesund, Norway
}

Correspondence should be addressed to Sigbjørn Berentsen; sigbjorn.berentsen@haugnett.no

Received 25 June 2014; Revised 6 November 2014; Accepted 10 November 2014

Academic Editor: Jerome Moreaux

Copyright (c) 2015 S. Berentsen and T. Sundic. This is an open access article distributed under the Creative Commons Attribution License, which permits unrestricted use, distribution, and reproduction in any medium, provided the original work is properly cited.

\begin{abstract}
Autoimmune hemolytic anemia (AIHA) is a collective term for several diseases characterized by autoantibody-initiated destruction of red blood cells (RBCs). Exact subclassification is essential. We provide a review of the respective types of AIHA with emphasis on mechanisms of RBC destruction, focusing in particular on complement involvement. Complement activation plays a definitive but limited role in warm-antibody AIHA (w-AIHA), whereas primary cold agglutinin disease (CAD), secondary cold agglutinin syndrome (CAS), and paroxysmal cold hemoglobinuria $(\mathrm{PCH})$ are entirely complement-dependent disorders. The details of complement involvement differ among these subtypes. The theoretical background for therapeutic complement inhibition in selected patients is very strong in CAD, CAS, and PCH but more limited in w-AIHA. The optimal target complement component for inhibition is assumed to be important and highly dependent on the type of AIHA. Complement modulation is currently not an evidence-based therapy modality in any AIHA, but a number of experimental and preclinical studies are in progress and a few clinical observations have been reported. Clinical studies of new complement inhibitors are probably not far ahead.
\end{abstract}

\section{Introduction}

Autoimmune hemolytic anemia (AIHA) is a heterogeneous group of disorders characterized by autoantibody-mediated destruction of red blood cells (RBCs) [1-3]. AIHA can be classified as shown in Table 1. Correct subclassification and identification of any underlying or associated disorder are critical for understanding the pathogenesis and for optimal therapeutic management [3-5].

The knowledge of etiology and pathogenesis, including details of RBC breakdown, is rapidly growing [3-7]. During the last five decades we have learned a great deal about the essential role of complement in subgroups of AIHA [68]. This insight is still expanding and possible therapeutic options for complement modulation are being explored [911]. Furthermore, though paroxysmal nocturnal hemoglobinuria $(\mathrm{PNH})$ is not an autoimmune disorder, the entirely complement-dependent pathogenesis and the success of therapeutic complement inhibition in this disease make it possible to learn lessons from $\mathrm{PNH}$ that might prove useful in treating AIHA [12].

In this review, we will address the pathogenetic mechanisms of AIHA, focusing in particular on the role of complement for RBC destruction and possible implications for the potential therapeutic use of complement modulators. Established therapies will be briefly mentioned since they have relevance for future therapeutic perspectives. Diagnostic procedures will not be described as such; comprehensive guidelines for diagnosis can be found elsewhere in the literature $[4,5]$.

\section{Warm-Antibody Autoimmune Hemolytic Anemia}

2.1. Etiology, Pathogenesis, and Associated Disorders. The incidence of AIHA has been estimated to be about 1:100 000 
TABLE 1: Autoimmune hemolytic anemia.

\begin{tabular}{l} 
Warm-antibody type \\
Primary \\
Secondary \\
Cold-antibody type \\
Primary chronic cold agglutinin disease \\
Secondary cold agglutinin syndrome \\
Associated with malignant disease \\
Acute, infection-associated \\
Paroxysmal cold hemoglobinuria \\
Mixed cold- and warm-antibody type \\
\hline
\end{tabular}

per year in adults [13] and even lower in children. Warmantibody AIHA (w-AIHA) accounts for approximately $75 \%$ of the cases $[1,2]$. The autoantibodies in w-AIHA have temperature optimum at $37^{\circ} \mathrm{C}$ and are invariably polyclonal, even when w-AIHA complicates a clonal B-cell lymphoproliferative disorder $[14,15]$. A general dysregulation of the immune system with impaired distinction between self and nonself seems essential to pathogenesis; the T-cell mediated regulation of the humoral immune system has been shown to play a critical role $[15,16]$. Polymorphism of the gene for the signal substance CTLA-4, which activates regulatory $\mathrm{T}$-cells $\left(\mathrm{T}_{\text {reg }}\right.$-cells), seems to bring about a disposition for autoimmunity [16]. $\mathrm{CD} 4^{+} \mathrm{CD} 25^{+} \mathrm{T}_{\text {reg }}$-cells are important for immunological tolerance and, thereby, for preventing wAIHA and other polyclonal autoimmune disorders [16].

On this background it is not surprising that a large number of immunological and lymphoproliferative disorders can be associated with w-AIHA. Secondary AIHA, that is, cases with a demonstrable associated or underlying disease, accounts for about $50 \%$ of w-AIHA, while the remaining $50 \%$ are classified as primary. The most frequently occurring associated lymphoproliferative disease is chronic lymphatic leukemia (CLL), whereas w-AIHA complicating another non-Hodgkin's lymphoma (NHL) is less common $[1,2,14]$. Examples of immunological disorders that can be associated with w-AIHA are systemic lupus erythematosus, rheumatoid arthritis, Sjögren's syndrome, primary biliary cirrhosis, hypothyroidism, inflammatory bowel disease, immune thrombocytopenia, and primary hypogammaglobulinemia $[1,2,15,17]$. Some patients have several associated diseases at the same time.

Autoantibody or complement fragment deposition on the $\mathrm{RBC}$ can be detected using polyspecific and monospecific direct antiglobulin test (DAT). The findings by monospecific DAT reflect, although not to a completely reliable extent, which immunoglobulin class(es) or complement fragments are present on the RBC surface. The autoantibodies in $\mathrm{w}$ AIHA are of the immunoglobulin G (IgG) class in most cases [4]. In up to $50 \%$ of w-AIHA, DAT is positive for complement fragments, most often C3d and usually in combination with IgG. IgA autoantibodies occur in $15-20 \%$ of the patients, either in combination with IgG or, more rarely, alone [18]. Cases with $\operatorname{IgA}$ as the sole autoantibody class may be misdiagnosed because reagents used in the polyspecific DAT do not usually contain anti-IgA. Warm autoantibodies of the IgM class have been assumed to be rare. Their frequency remains somewhat controversial, however, because they may have low affinity to the antigen and may have detached from the RBC surface before they can be detected by DAT $[19,20]$.

In $3-10 \%$ of patients with w-AIHA, DAT is found to be negative $[4,21]$. The most established explanation is IgG deposition on RBC below the sensitivity threshold for DAT or, less frequently, occurrence of IgA as the only autoantibody class $[4,18]$. The hypothesis that T- or NK-cell mediated immunity can destroy erythrocytes without involving the humoral immune system has been supported by a few casuistic and experimental observations [6, 22]. Furthermore, some evidence has been provided that $\mathrm{Fc} \gamma \mathrm{RI}$ receptors on macrophages bind monomeric serum-IgG which can include low-concentration specific RBC antibodies. This will create "armed macrophages" carrying RBC antibodies whose Fab portion can react with nonsensitized RBC [23]. "DATnegative AIHA" represents a diagnostic challenge. Elution techniques and flow cytometric methods may be of some value but in clinical practice, often, DAT-negative AIHA remains an exclusion diagnosis. The usefulness of newer gel centrifugation tests for IgG, IgG subclasses, and complement fragments in detecting autoimmune pathogenesis of hemolytic anemia was explored in a recent study. DAT strength remained the best diagnostic indicator for AIHA and had the strongest association with AIHA compared with other commercially available immunohematology tests [24].

2.2. Mechanisms of Erythrocyte Destruction. RBCs coated with warm-reactive autoantibodies are sequestered and phagocytosed by macrophages, primarily in the spleen [2527]. The macrophage surface expresses receptors for the $\mathrm{Fc}$ region of the immunoglobulin molecules, which enables trapping and ingestion of the opsonized RBCs [28, 29]. Often, however, phagocytosis is incomplete and results in formation of spherocytes $[7,28]$. This has been explained in part by the removal of more membranes than volume. In addition, ectoenzymes on the macrophage surface cause microperforations of the RBC membrane, increasing its permeability and thereby promoting the transition from a biconcave to a spherical shape of the cell $[7,25,28]$. Spherocytes are prone to further destruction during subsequent passages through the spleen. The severity of hemolysis correlates with the degree of spherocytosis, but not with the strength of DAT positivity $[4,7,26]$.

On RBCs heavily coated with immunoglobulin, the amount of antigen-antibody complex can be sufficient for binding complement protein complex $\mathrm{Cl}$ and, thereby, for activation of the classical complement pathway (Figure 1) [30-32]. Unlike IgG, IgM is a potent complement activator but, as previously mentioned, is usually not found on the RBC surface by DAT in w-AIHA [19]. Regarding the IgG subclasses, IgG3 activates complement more efficiently than does IgG1, while IgG2 is a weak activator and there is no good evidence for complement activation by IgG4 $[33,34]$. IgA does not probably activate complement. Despite this, 


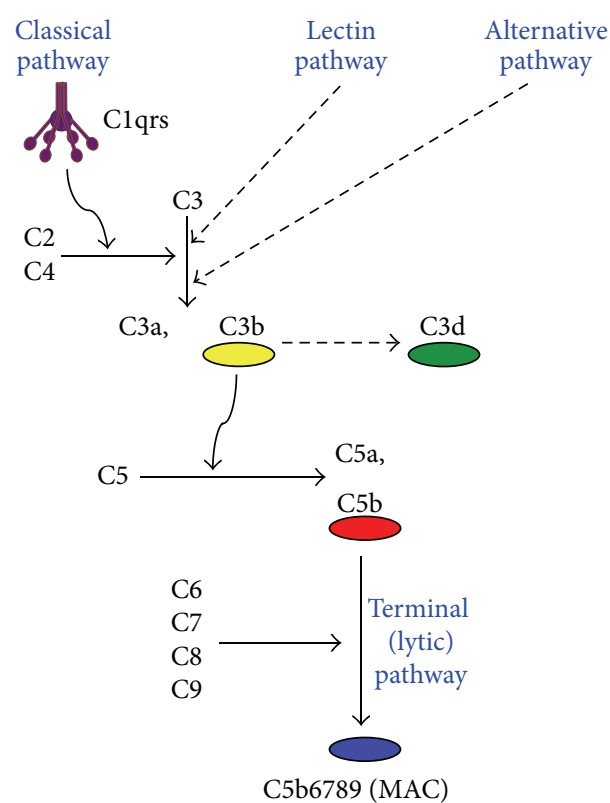

Figure 1: The complement cascade, simplified. Only components relevant for this paper are shown. The lectin and alternative pathways, converging with the classical pathway at the C3 activation level, are indicated but not shown in detail. Physiological inhibitors and positive feedback loops are not shown. C: complement protein; MAC: membrane attack complex.

however, IgA-deposition on RBC can lead to fulminant hemolysis [18, 35]. A probable explanation is involvement of $\operatorname{IgM}$ even in some cases where only IgG or IgA is detected, since IgM will often detach from the RBC before it can be detected by DAT [20]. At least in IgA-induced hemolysis, hemagglutination per se has also been shown to play a role. Upon complement activation in w-AIHA, phagocytosis of C3b-opsonized erythrocytes by Kupfer cells in the liver is responsible for most of the RBC destruction, while fullblown intravascular hemolysis mediated by the terminal complement pathway is usually not prominent $[4,7,31]$. The explanation for this is probably the modest activation of the complement pathway, combined with the protective effect of the physiological cell surface complement inhibitors CD55 and $\mathrm{CD} 59$ which, unlike in $\mathrm{PNH}$, are intact in AIHA.

The pathways of RBC destruction in w-AIHA are summarized in Figure 2. In conclusion, complement activation does occur to some extent, at least in a proportion of the patients, but is hardly essential for hemolysis in w-AIHA. DAT positivity for $\mathrm{C} 3$ fragments is a marker of complement involvement.

2.3. Therapy. The cornerstone of established pharmacological therapy for w-AIHA is unspecific immunosuppression. Corticosteroids remain first-line treatment. However, high initial doses are required, responses are often achieved slowly, and the rate of sustained remissions following weaning of steroids is only $15-30 \%$ unless second-line therapy is administered $[3,4,36]$. Combination with rituximab in the first-line setting has been shown to significantly increase

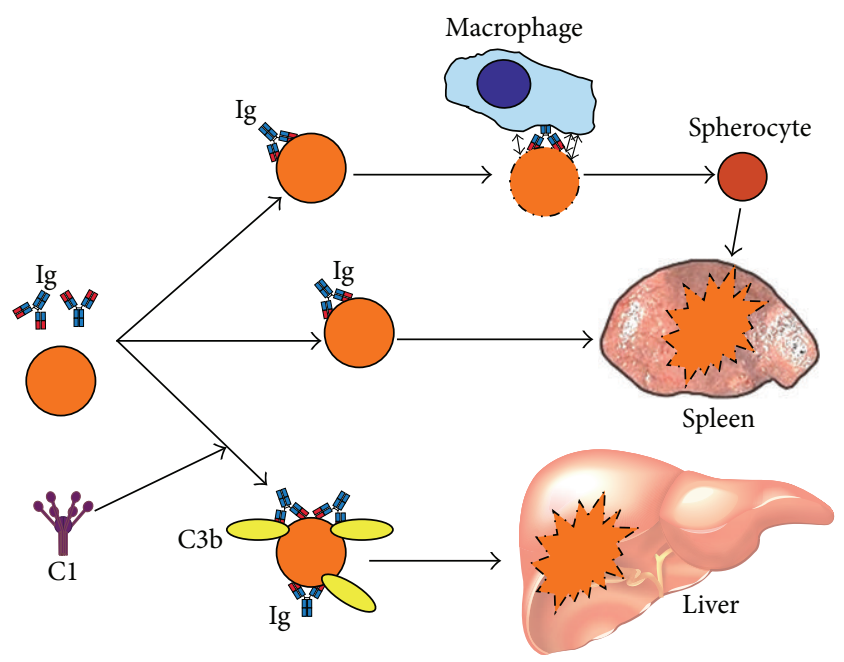

FIGURE 2: Mechanisms of erythrocyte destruction in warmantibody autoimmune hemolytic anemia. Ig: immunoglobulin; C: complement protein.

response rate and duration [36], although rituximab has not gained general acceptance by most authors as part of firstline therapy [37]. The concern of adverse effects of rituximab in the AIHA setting will be discussed below in the context of therapy for CAD.

Since most RBC destruction occurs in the spleen, it is not surprising that splenectomy is a reasonably efficient secondline treatment, resulting in response in about two-thirds of the patients [4]. An alternative, safe, and efficient second-line option, if not used as part of combination therapy in the first line, is infusions of rituximab [38]. In the third-line situation, one may use immunosuppressive drugs such as danazol, azathioprine, cyclophosphamide, or cyclosporine $[3,4,39]$, although response rates are poorly documented and most publications are case reports or small retrospective series. High-dose cyclophosphamide or alemtuzumab has been used with success in refractory cases [37]. In w-AIHA secondary to lymphoproliferative diseases, therapy for the underlying disorder is often essential and should be considered at an early stage. The future possibilities of complement-directed therapy will be addressed below. Transfusion in w-AIHA is an important and complex issue which has been comprehensively discussed elsewhere $[4,40,41]$. To avoid severe transfusion reactions and alloimmunization with increasing transfusion problems, indications should be restrictive and specific precautions undertaken. The risk of alloimmunization should be reduced by extended blood group phenotyping or the use of prophylactic antigen-matched donor blood and a bed-side biological compatibility test should be performed $[40,42,43]$.

\section{Primary Chronic Cold Agglutinin Disease}

3.1. Etiology and Pathogenesis. We should distinguish between primary cold agglutinin disease (CAD) and secondary cold agglutinin syndrome (CAS) [5]. As will 
be further explained below, CAD is a precisely defined clinicopathological entity and should, therefore, be called a disease, not syndrome [44]. Secondary CAS, on the other hand, is a syndrome complicating a variety of infectious and neoplastic disorders, not a well-defined disease. In a Norwegian population-based study, the prevalence of CAD was 16 per million and the incidence was about 1 per million per year, making CAD account for approximately $15 \%$ of AIHA $[1,2,45]$.

Cold agglutinins (CA) are autoantibodies that agglutinate RBCs with a temperature optimum of $3-4^{\circ} \mathrm{C}$ but may also act in a warmer environment, depending on the thermal amplitude of the CA $[5,46]$. If the thermal amplitude exceeds $28-30^{\circ} \mathrm{C}$, the CA will be pathogenic. Low-affinity CA also occur in many healthy individuals; these nonpathogenic CA are polyclonal, have low thermal amplitude, and are present in low titers, not higher than 256 and usually lower than 64 . More than $90 \%$ of pathogenic CA are of the IgM class and these IgM macromolecules can be pentameric or hexameric $[45,47,48]$.

In general, monoclonal $\mathrm{CA}$ are more pathogenic than polyclonal CA and hexameric IgM is more pathogenic than pentameric IgM $[5,48,49]$. It has been known for decades that, in patients with CAD, IgM-antibodies with CA-activity are monoclonal and, in more than $90 \%$ of the patients, show $\kappa$ light chain restriction [50]. Accordingly, CAD patients must have a clonal B-cell lymphoproliferative disorder which has not been fully elucidated until the last years. Two large, retrospective studies of consecutive patients with primary CAD found signs of a bone marrow clonal lymphoproliferation in most patients, but in both series the individual hematological and histological diagnoses showed a striking heterogeneity $[45,51]$. In one of the series, lymphoplasmacytic lymphoma (LPL) was the most frequent finding, while marginal zone lymphoma (MZL), unclassified clonal lymphoproliferation, and reactive lymphocytosis were also frequently reported [45]. The explanation for this perceived heterogeneity was probably revealed by a recent study in which bone marrow biopsy samples and aspirates from 54 patients with CAD were systematically reexamined by a group of lymphoma pathologists, using a standardized panel of morphological, immunohistochemical, flow cytometric, and molecular methods [44]. The bone marrow findings in these patients were consistent with a surprisingly homogeneous disorder termed "primary CA-associated lymphoproliferative disease" by the authors and distinct from LPL, MZL, and other previously recognized lymphoma entities. The MYD88 L265P somatic mutation, typical for LPL, could not be detected in the samples from patients with CAD $[44,52]$.

3.2. Mechanisms of Erythrocyte Destruction. CA are usually directed against the Ii blood group system, most CA in CAD being specific for the I carbohydrate antigen [53-55]. Cooling of blood during passage through acral parts of the circulation allows $\mathrm{CA}$ to bind to RBC and cause agglutination (Figure 3). Being a strong complement activator, antigenbound IgM-CA on the cell surface binds $\mathrm{Cl}$ and thereby initiates the classical complement pathway $[8,56,57]$. $\mathrm{Cl}$

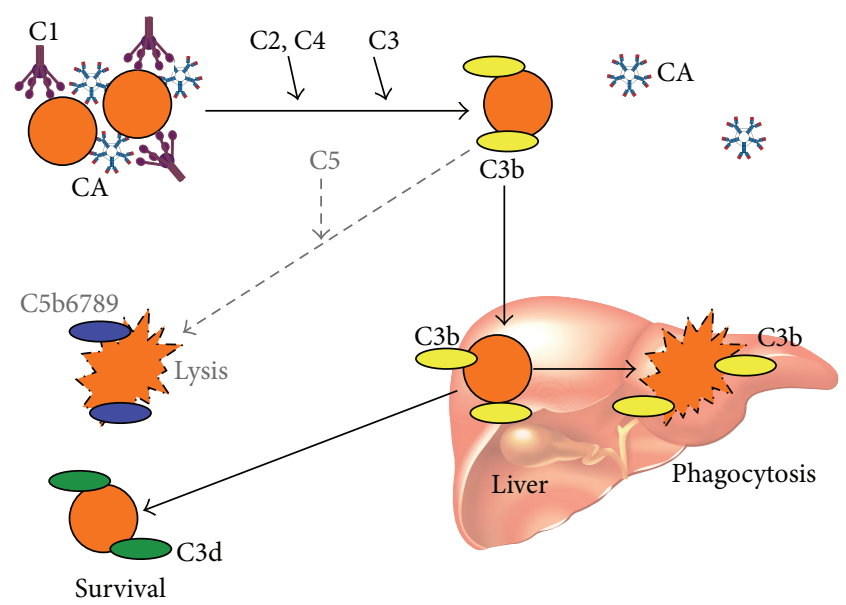

FIGURE 3: Immune-initiated, complement-mediated erythrocyte destruction in cold agglutinin disease (CAD) and cold agglutinin syndrome (CAS). See text for further explanation. Black arrows: major pathway; gray/dotted arrows: minor pathway; CA: cold agglutinin; C: complement protein.

esterase activates $\mathrm{C} 4$ and $\mathrm{C} 2$, generating $\mathrm{C} 3$ convertase, which results in the cleavage of $\mathrm{C} 3$ to $\mathrm{C} 3 \mathrm{a}$ and $\mathrm{C} 3 \mathrm{~b}$. Upon returning to central parts of the body with a temperature of $37^{\circ} \mathrm{C}$, IgM-CA detaches from the cell surface, allowing agglutinated erythrocytes to separate from each other, while C3b remains bound. A proportion of the C3b-coated RBCs is sequestered by macrophages of the reticuloendothelial system, mainly Kupfer cells in the liver. On the surface of the surviving RBCs, C3b is cleaved, leaving high numbers of C3d molecules on the cell surface. These mechanisms explain why the monospecific DAT is strongly positive for C3d in patients with CA-mediated hemolysis and, in the majority, negative for IgM and IgG [45].

Complement activation may proceed beyond the $\mathrm{C} 3 \mathrm{~b}$ formation step, resulting in C5 activation, formation of the membrane attack complex (MAC), and intravascular hemolysis. Due to surface-bound regulatory proteins such as CD55 and CD59, however, the complement activation is usually not sufficient to produce clinically significant activation of the terminal complement pathway. The major mechanism of hemolysis in stable disease, therefore, is the extravascular destruction of C3b-coated erythrocytes [10, 31, 57]. Obviously, however, C5-mediated intravascular hemolysis does occur in severe acute exacerbations and in some profoundly hemolytic patients, as evidenced by the finding of hemoglobinuria in $15 \%$ of the patients and the observation of a beneficial effect of $\mathrm{C} 5$ inhibition in at least occasional patients $[45,51,58,59]$.

Febrile infections, major trauma, or major surgery can result in acute exacerbation of hemolytic anemia in at least two-thirds of patients with CAD $[45,58,60]$. The explanation for this paradoxical exacerbation is that, during steady-state chronic disease, most patients are complement-depleted with low levels of C3 and often undetectable levels of C4. During acute phase reactions, C3 and C4 are repleted and exacerbation of complement-induced hemolysis ensues $[55,58]$. 
3.3. Therapy. In textbooks and review articles, it is often postulated that typical patients with CAD are just slightly anemic and do not need pharmacological therapy. This holds true for a minority only; the median hemoglobin level in affected individuals is $9.0 \mathrm{~g} / \mathrm{dL}$ and the lower tertile is $8.0 \mathrm{~g} / \mathrm{dL}$ [45]. Furthermore, at least $90 \%$ of the patients experience coldinduced circulatory symptoms caused by RBC agglutination, most often in the form of acrocyanosis and/or Reynaudphenomena that can range from slight to disabling [45]. In many patients, therefore, CAD is not an indolent disease in terms of major clinical symptoms and quality of life. In Norway as well as the United States, drug therapy had been attempted in $70-80 \%$ of unselected patients studied in two relatively large retrospective series $[45,51]$. In contrast to $\mathrm{W}$ AIHA, corticosteroids and other unspecific immunosuppressive drugs are of little or no value in CAD [45, 61].

The relative success in therapy for CAD during the last 10-12 years has been achieved by targeting the pathogenic Bcell clone [62]. Rituximab monotherapy has been shown in prospective studies to induce remission in approximately half of the patients, although complete remissions are unusual and the median response duration is only about 1 year $[63,64]$. In both studies, events of cytokine-related reactions to rituximab were few and readily treatable. The studies found no significant problems with infectious complications due to the induced B-cell lymphopenia and hypogammaglobulinemia $[45,63,64]$. Data from rituximab maintenance in follicular lymphoma indicate that, in adults, even prolonged or repeated administration is safe with regard to infections [65]. Very rare cases of progressive multifocal leukoencephalopathy and hepatitis B reactivation have been reported, however, in patients receiving rituximab for polyclonal autoimmune disorders. Any causal associations are uncertain because of concomitant immunosuppressive therapies and immune dysregulation as part of the autoimmune disease itself [66].

In a more recent prospective trial, combined therapy with rituximab and fludarabine produced very high response rates (remission in $75 \%$ of the patients, including $20 \%$ complete remissions) and the median response duration was more than 66 months [67]. This regimen was, however, found to be significantly more toxic than rituximab monotherapy. No other immunochemotherapy regimens have been studied in published clinical trials. According to single case reports, favorable outcome has been observed following bortezomibbased regimens [68] and rituximab-bendamustine combination therapy [69].

Transfusion can safely be given in CAD provided specific precautions are undertaken, although these precautions are entirely different from those required in w-AIHA. Such requirements have been extensively described elsewhere [5, 70]. The perspective for future therapeutic use of complement inhibitors will be addressed below.

\section{Secondary Cold Agglutinin Syndrome}

Secondary CAS is far more uncommon than primary CAD. Among 295 consecutive individuals with AIHA described retrospectively by Dacie in a single-center series, 7 patients
(2.4\%) were classified as having CAS secondary to malignant disease [1]. CAS has been described in patients diagnosed with diffuse large B-cell lymphoma, Hodgkin's lymphoma, carcinomas, sarcomas, metastatic melanoma, and chronic myeloproliferative disorders [2]. Some of these associations have been poorly documented [5], and the most convincing association with malignant disease has been described with non-Hodgkin's lymphoma [71-74]. In CAS complicating aggressive lymphoma, the CA are monoclonal, most often IgM, and have anti-I specificity. In contrast to CA found in primary $\mathrm{CAD}$, however, the light chain restriction can be $\lambda$ as well as $\kappa[71,74]$.

Polyclonal anti-I specific CA of the IgM class are produced as part of the physiological immune response in Mycoplasma pneumoniae pneumonia. They do not usually give rise to significant hemolysis. In occasional patients, however, production of high-titer, high-thermal amplitude CA results in hemolytic anemia which is transient but can be severe $[5,75,76]$. CAS complicating Mycoplasma pneumoniae infection has been reported to account for approximately $8 \%$ of AIHA [1]. Still more uncommon but less severe, polyclonal anti-i specific CA of the IgM or IgG class can result in CAS in Epstein-Barr virus infection [5, 77]. Transient CAS has also been described following cytomegalovirus infection, varicella, rubella, adenovirus infection, influenza A, Legionella pneumophila pneumonia, listeriosis, and pneumonia caused by Chlamydia species [5].

In CAS secondary to infection or aggressive lymphoma, the RBC breakdown is complement-dependent, mediated by exactly the same mechanisms as in primary CAD (Figure 3) $[5,7]$. Treatment of the underlying disease, if relevant and available, is often the only possible drug therapy for the hemolytic complication. Corticosteroid therapy has been used but is not evidence-based. In severely anemic patients, transfusions can safely be given provided the same precautions are carefully observed as in primary CAD [5].

\section{Paroxysmal Cold Hemoglobinuria}

In paroxysmal cold hemoglobinuria $(\mathrm{PCH})$, polyclonal coldreactive IgG-antibodies bind to the $\mathrm{RBC}$ surface protein antigen termed $\mathrm{P}$ but do not agglutinate the erythrocytes. The resulting hemolysis is entirely complement-dependent and the temperature optimum for complement activation is at $37^{\circ} \mathrm{C}[78,79]$. Such biphasic antibodies are called DonathLandsteiner hemolysins. In Donath-Landsteiner's test, one sample of patient blood is incubated at $4^{\circ} \mathrm{C}$ and then at $37^{\circ} \mathrm{C}$, while another sample is incubated at $37^{\circ} \mathrm{C}$ without having been preincubated in the cold $[78,79]$. If biphasic autoantibodies are present, hemolysis will be observed only in the sample preincubated at $4^{\circ} \mathrm{C}$. The sensitivity is limited because the patient blood is often complement-depleted, and, in more sensitive modifications of the test, complement is added and/or papain pretreated RBCs are used [79].

Fifty to 100 years ago, PCH was associated with tertiary syphilis, but this form is hardly seen anymore. In the 21th century, $\mathrm{PCH}$ occurs almost exclusively in children and accounts for $1-5 \%$ of childhood AIHA, making it a rare disease [80]. 


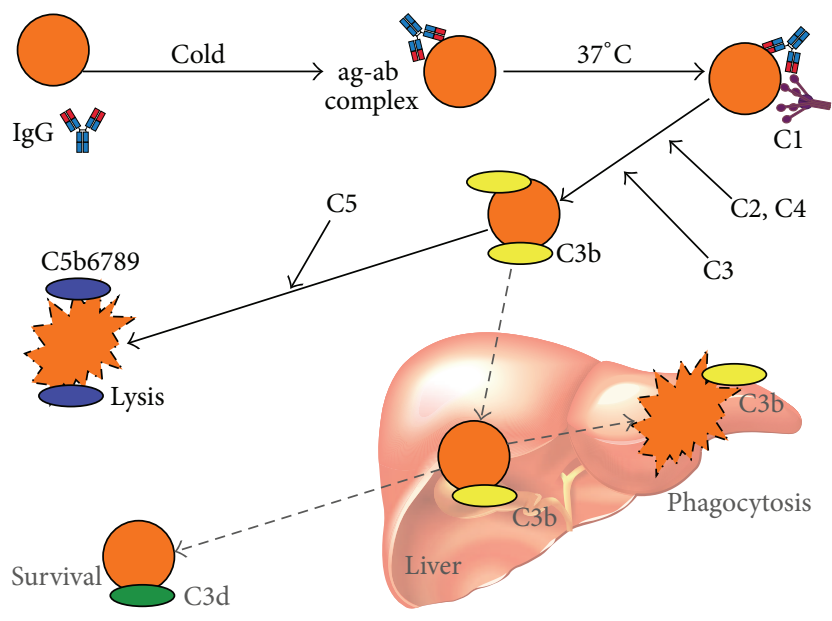

FIGURE 4: Immune-initiated, complement-mediated erythrocyte destruction in paroxysmal cold hemoglobinuria $(\mathrm{PCH})$ showing a biphasic temperature optimum. Black arrows: major pathway (intravascular hemolysis); gray/dotted arrows: possible minor pathway; Ig: immunoglobulin; ag: antigen; ab: antibody; C: complement protein.

It appears as an acute, postinfectious complication, in most cases following a virus infection [79]. Single cases have also been reported in Haemophilus influenzae infection and, recently, visceral leishmaniasis [80, 81].

The $\mathrm{P}$-anti-P complex is a very strong complement trigger, resulting in full-blown activation of the classical and terminal pathways (Figure 4). The hemolysis, therefore, is intravascular and massive; the onset is usually sudden and the clinical features include fever, pallor, jaundice, severe anemia, and macroscopic hemoglobinuria $[79,81]$. Even though $\mathrm{PCH}$ is a transient complication with good prognosis, most patients will need transfusions, which can safely be given provided the same precautions are undertaken as in other cold-antibody AIHA [5]. Apart from therapy for any treatable underlying infection, no disease-modifying intervention has been documented. Although the administration of corticosteroids has been followed by improvement in some reported cases, the effect remains anecdotal and unproven $[79,81]$.

\section{Mixed-Type Autoimmune Hemolytic Anemia}

Mixed warm- and cold-antibody AIHA is probably very rare. The diagnostic work-up is complex and the condition is supposed to be overdiagnosed [82]. There are two obvious sources of error. First, patients with w-AIHA can, like healthy individuals, produce low-titer, low-thermal amplitude CA of no clinical significance. Second, up to $20 \%$ of patients with CAD have IgG on the RBC surface in addition to C3d $[45,82]$.

\section{Complement Modulation for the Treatment of AIHA}

7.1. Available Substances and Experimental Studies. The potential of pharmacological complement modulation for the treatment of AIHA will depend on (a) the type of AIHA and extent and level of complement involvement, (b) the availability, safety, and efficacy of complement-modulating drugs, and (c) the specific level of complement inhibition by these drugs. The search for targeted, therapeutic inhibitors of the complement cascade has been going on for 30-40 years, with few examples of success so far [9]. New in vitro and in vivo models for testing the impact of specific complement inhibition on immune hemolysis are, however, still being developed [10, 83].

Plasma-derived or recombinant C1-esterase inhibitor (C1INH) has been available for decades and has been successfully used for the treatment of hereditary angioedema (HAE) [84]. Although not a complement-mediated disorder, HAE is caused by lack or deficiency of endogenous C1-INH and replacement therapy has been well studied. In AIHA, on the other hand, endogenous C1-INH production is normal, indicating that physiological concentrations of the inhibitor will not block complement-mediated hemolysis.

Eculizumab, a humanized monoclonal C5-antibody, has been shown to efficiently inhibit complement at the C5 level and, thereby, block the terminal pathway and prevent intravascular hemolysis by MAC. Therapy with eculizumab has been a great success in $\mathrm{PNH}$, although complementmediated hemolysis is not completely prevented [85]. The explanation for this is probably that patients with $\mathrm{PNH}$ lack physiological inhibitors both at a downstream level in the terminal pathway (CD59) and at an upstream level in the classical pathway (CD55). In consequence, a slight to moderate hemolysis mediated by phagocytosis of C3bopsonized RBC will still occur along the same pathway as described in CAD, independent of C5 activation or inhibition [12].

Some newer complement-modulating drugs have been studied with promising results in preclinical experiments but not yet in the in vivo setting. Compstatin Cp40 is a lowmolecular weight peptide complement inhibitor that blocks cleavage of $\mathrm{C} 3$ and has been found to efficiently prevent hemolysis of RBC from $\mathrm{PNH}$ patients in vitro [86].

TNT003, a mouse monoclonal anti-C1s antibody (Figure 5), has recently been shown to completely inhibit in vitro hemolysis induced by CA [10]. This antibody targets $\mathrm{Cls}$ serine protease activity. Using CA samples from 40 patients with CAD, the authors found that TNT003 prevented CA-induced deposition of C3 fragments on the $\mathrm{RBC}$ at the same concentration of antibody that stopped hemolysis. Furthermore, C1s inhibition by TNT003 resulted in prevention of in vitro erythrophagocytosis by a phagocytic cell line. The classical-pathway-driven production of anaphylotoxins C4a, C3a, and C5a was also inhibited [10].

7.2. Complement Inhibition in Subtypes of AIHA: Future Perspective. In w-AIHA, as described above, complement activation plays some role but is not essential for pathogenesis in most patients. Complement modulation may be expected, therefore, to be of limited therapeutic value in w-AIHA in general and of no value if DAT is negative for C3 fragments. According to a recent, well-described case report, however, 


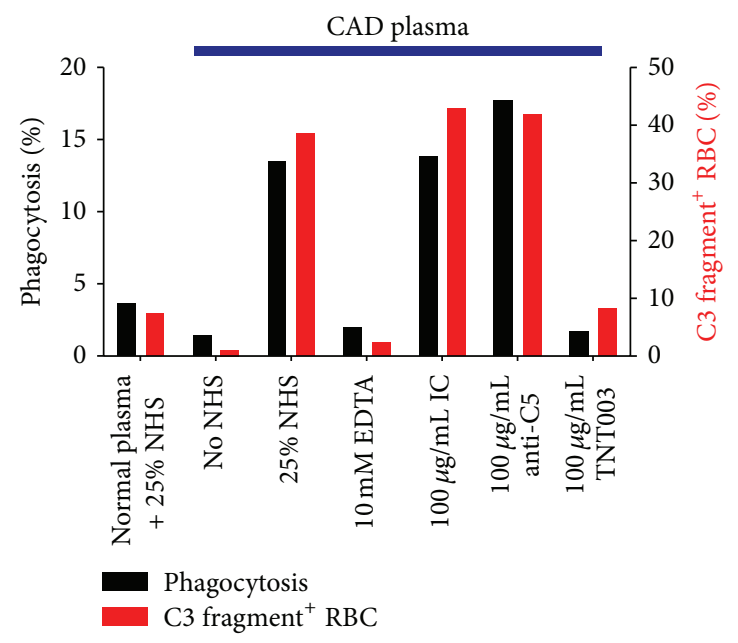

(a)

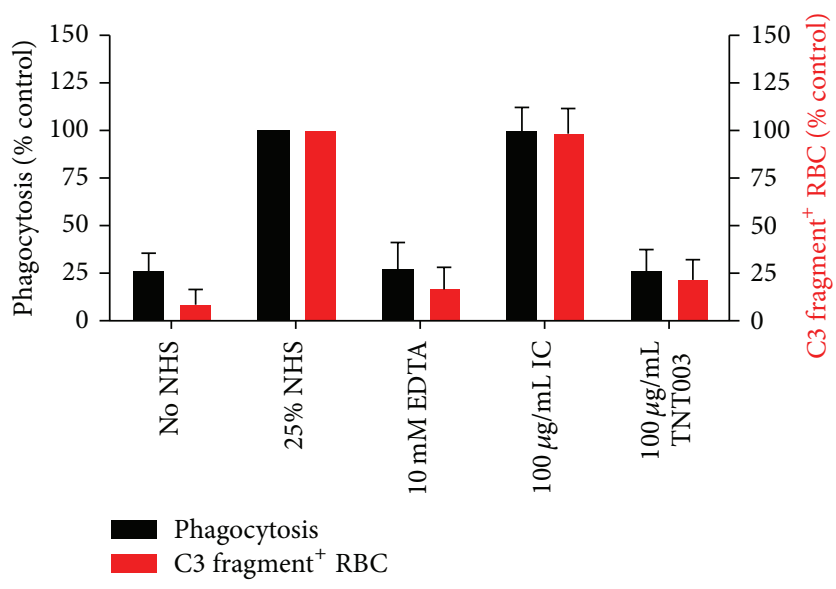

(b)

FIGURE 5: In vitro effects of anti-C1s antibody TNT003 on phagocytosis (black columns, left $y$-axis) and complement fragment deposition (red columns, right $y$-axis) on red blood cells after addition of normal human plasma and cold agglutinin-rich plasma (under blue bar), respectively. TNT003 inhibits phagocytosis and complement deposition, while anti-C5 has no impact. CAD: primary chronic cold agglutinin disease; NHS: normal human serum; IC: isotype control. Reproduced from Blood (Shi et al. 2014, [10]) with permission. Copyright: Blood, the Journal of the American Society of Hematology.

Wouters and colleagues observed favorable effect of plasmaderived C1-INH in a patient with a C3d-positive, therapyresistant severe w-AIHA secondary to an aggressive nonHodgkin's lymphoma [87]. Although very high doses of $\mathrm{Cl}$-INH were required, hemolysis was efficiently controlled and the efficacy of RBC transfusion dramatically improved following treatment. No other clinical observations on the results of complement inhibition have been published in wAIHA. In patients with a positive DAT for C3d and very severe hemolysis, further studies of complement inhibition even at a more downstream level would be of interest, mainly as an attempt to temporarily control hemolysis.

Given that hemolysis in CAD is entirely complementdependent, studies of complement inhibition would be relevant in CAD. A case report by Röth and coauthors described favorable effect of therapy with eculizumab [59]. This observation may seem somewhat surprising, since the predominant hemolytic pathway in CAD is not C5/MACmediated. A probable explanation is that activation of the terminal complement pathway does occur, after all, in acute exacerbations, in the chronic state of some severely affected patients and, possibly, as a minor pathway also in less severely affected patients. Further studies will be of interest.

In theory, complement inhibition at the $\mathrm{Cl}$ level should be very promising in CAD because this will block the classicalpathway-dependent, C3b-mediated extravascular hemolysis without compromising the alternative and lectin complement pathways. The published in vitro study of TNT003 is highly interesting, therefore, and it is to be hoped that a corresponding humanized antibody can be developed and further tested in the preclinical and clinical setting $[10,11]$.

Given that immunochemotherapy directed at the pathogenic B-cell clone is efficient and requires administration only for a limited period of time, do we actually need complement-modulating therapies for CAD? First, in at least
$25 \%$ of the patients, immunochemotherapy is unsuccessful because of treatment failure or toxicity [67]. Second, rapidly acting therapies should be developed for some specific clinical situations, for example, acute severe exacerbations induced by infections, trauma, or major surgery and, possibly, before cardiac surgery in selected patients.

In the uncommon cases of CAS secondary to specific infection, there is often no need for therapy for the CAS per se. However, this is not always the case. Particularly in CAS following Mycoplasma pneumoniae pneumonia, the patients can be profoundly anemic and transfusion dependent for weeks until spontaneous resolution occurs [5]. Clinicians and patients would welcome a possibility for temporary control of this situation by complement inhibition along the same lines that may be developed in primary CAD. Systematic studies would be interesting but probably difficult to perform because of the rarity of the disorder.

In the rare cases of postinfectious $\mathrm{PCH}$ in children, measures for temporary control of the hemolysis will be valuable if such therapies can be developed. Since the terminal complement pathway is heavily involved, we do not necessarily need new substances; exploring the efficacy of eculizumab would be of great interest. Probably, however, prospective trials will never be performed because there are too few patients for such studies.

Present and future possibilities for therapeutic complement inhibition in AIHA are summarized in Figure 6. It is important to ask whether such therapy will be dangerous. The complement system is, after all, an essential part of the innate immune system. Based on studies of eculizumab in $\mathrm{PNH}$, we already have extensive information on the risk of severe infection following C5 inhibition. Provided the patients can be efficiently protected against meningococci, studies and clinical experience have shown that the risk of infection is negligible [85]. Complement inhibition at the C3 


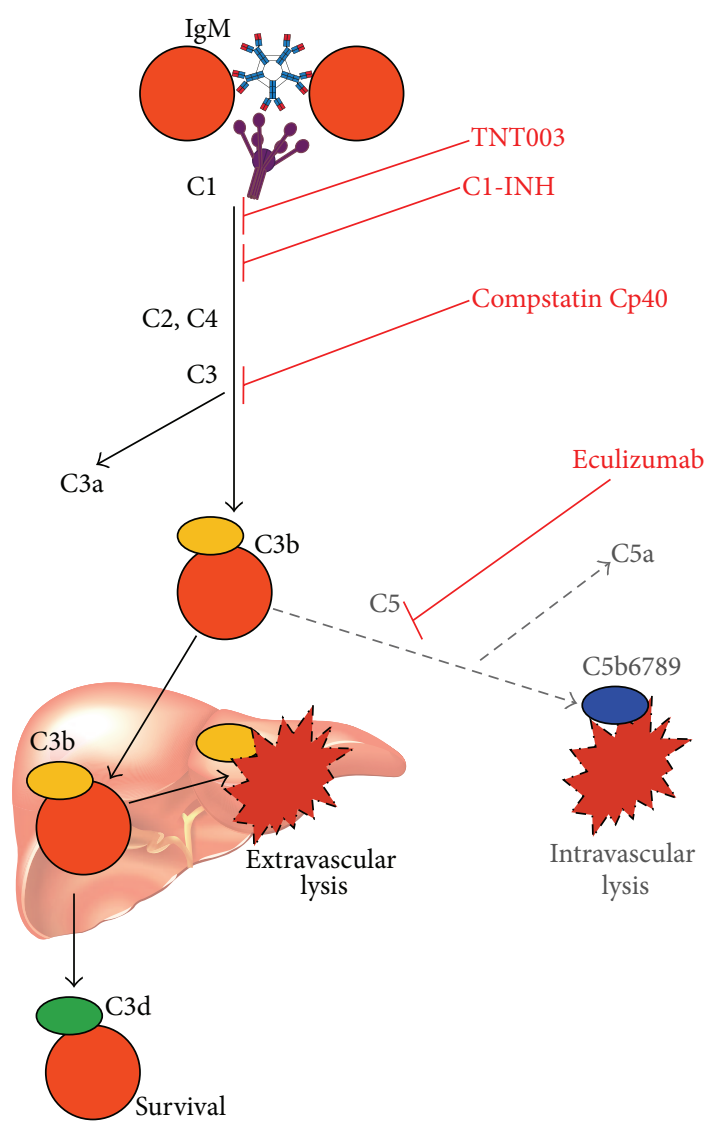

FIGURE 6: Future perspective on complement modulation in AIHA. Inhibitors and levels of inhibition. Black arrows: major pathway in cold agglutinin disease (CAD); gray/dotted arrows: minor pathway in CAD and major pathway in paroxysmal cold hemoglobinuria; Ig: immunoglobulin; C: complement protein; TNT003: mouse monoclonal C1s antibody; C1-INH: C1 esterase inhibitor. Previously published in Blood (Berentsen 2014, [11]), reused with permission. Copyright: Blood, the Journal of the American Society of Hematology.

level may carry a much higher risk because efficient inhibition of C3 will completely block complement activation beyond this level, whether initiated by the classical, alternative, or lectin pathway $[11,86]$. Interestingly, however, the still more proximal blockade at the $\mathrm{C} 1$ level achieved by TNT003 will selectively affect the classical pathway as required for control of hemolysis in CAD, while the lectin and alternative pathways will remain intact. Probably, therefore, these pathways will still enable the system to generate anaphylotoxins C3a and $\mathrm{C} 5 \mathrm{a}$ in response to microbial stimuli, even though the production of these anaphylotoxins induced by the classical pathway will be blocked $[10,11]$. Although this selectivity may, theoretically, reduce the risk of infection, careful studies will be required to address this issue.

\section{Conclusion}

The mechanism of RBC destruction differs considerably among the various types of AIHA, and so do the extent, level, and details of complement involvement. The theoretical background for therapeutic complement inhibition in selected subgroups of patients is very strong in CAD, CAS, and $\mathrm{PCH}$ but more limited in w-AIHA. Complement modulation is not an established or evidence-based therapy modality in any type of AIHA, but a number of experimental and preclinical studies are in progress and a few clinical observations have been reported. It will be important to carefully address safety issues. Clinical studies of new complement inhibitors are probably not far ahead.

\section{Conflict of Interests}

Tatjana Sundic declares no conflict of interests.

\section{Acknowledgment}

Sigbjørn Berentsen has received financial research support from Mundipharma and travel grants and lecture honoraria from Alexion.

\section{References}

[1] J. Dacie, "The auto-immune haemolytic anaemias: introduction," in The Haemolytic Anaemias, J. Dacie, Ed., vol. 3, pp. 1-5, Churchill Livingstone, London, UK, 1992.

[2] R. J. Sokol, S. Hewitt, and B. K. Stamps, "Autoimmune haemolysis: an 18-year study of 865 cases referred to a regional transfusion centre," British Medical Journal, vol. 282, no. 6281, pp. 2023-2027, 1981.

[3] M. Michel, "Classification and therapeutic approaches in autoimmune hemolytic anemia: an update," Expert Review of Hematology, vol. 4, no. 6, pp. 607-618, 2011.

[4] C. H. Packman, "Hemolytic anemia due to warm autoantibodies," Blood Reviews, vol. 22, no. 1, pp. 17-31, 2008.

[5] S. Berentsen and G. E. Tjønnfjord, "Diagnosis and treatment of cold agglutinin mediated autoimmune hemolytic anemia," Blood Reviews, vol. 26, no. 3, pp. 107-115, 2012.

[6] G. Garratty, “The James Blundell Award Lecture 2007: do we really understand immune red cell destruction?" Transfusion Medicine, vol. 18, no. 6, pp. 321-334, 2008.

[7] J. Dacie, "Auto-immune haemolytic anaemia (AIHA): pathogenesis," in The Haemolytic Anaemias, J. Dacie, Ed., vol. 3, pp. 392-451, Churchill Livingstone, London, UK, 1992.

[8] S. Berentsen, K. Beiske, and G. E. Tjønnfjord, "Primary chronic cold agglutinin disease: an update on pathogenesis, clinical features and therapy," Hematology, vol. 12, no. 5, pp. 361-370, 2007.

[9] E. L. Larghi and T. S. Kaufman, "Modulators of complement activation: a patent review (2008-2013)," Expert Opinion on Therapeutic Patents, vol. 24, no. 6, pp. 665-686, 2014.

[10] J. Shi, E. L. Rose, A. Singh et al., "TNT003, an inhibitor of the serine protease C1s, prevents complement activation induced by cold agglutinin disease patient autoantibodies," Blood, vol. 123, no. 26, pp. 4015-4022, 2014.

[11] S. Berentsen, "Complement, cold agglutinins, and therapy," Blood, vol. 123, no. 26, pp. 4010-4012, 2014.

[12] A. Hill, R. P. Rother, L. Arnold et al., "Eculizumab prevents intravascular hemolysis in patients with paroxysmal nocturnal 
hemoglobinuria and unmasks low-level extravascular hemolysis occurring through $\mathrm{C}_{3}$ opsonization," Haematologica, vol. 95 , no. 4, pp. 567-573, 2010.

[13] B. C. Gehrs and R. C. Friedberg, "Autoimmune hemolytic anemia," American Journal of Hematology, vol. 69, no. 4, pp. 258-271, 2002.

[14] L. F. Diehl and L. H. Ketchum, "Autoimmune disease and chronic lymphocytic leukemia: autoimmune hemolytic anemia, pure red cell aplasia, and autoimmune thrombocytopenia," Seminars in Oncology, vol. 25, no. 1, pp. 80-97, 1998.

[15] E. Fagiolo, "Immunological tolerance loss vs. erythrocyte self antigens and cytokine network disregulation in autoimmune hemolytic anaemia," Autoimmunity Reviews, vol. 3, no. 2, pp. 53-59, 2004.

[16] F. J. Ward, A. M. Hall, L. S. Cairns et al., "Clonal regulatory $\mathrm{T}$ cells specific for a red blood cell autoantigen in human autoimmune hemolytic anemia," Blood, vol. 111, no. 2, pp. 680687,2008

[17] P. C. Hoffman, "Immune hemolytic anemia-selected topics," Hematology. American Society of Hematology. Education Program, pp. 13-18, 2006.

[18] D. Janvier, F. Sellami, F. Missud et al., "Severe autoimmune hemolytic anemia caused by a warm IgA autoantibody directed against the third loop of band 3 (RBC anion-exchange protein 1).," Transfusion, vol. 42, no. 12, pp. 1547-1552, 2002.

[19] P. A. Arndt, R. M. Leger, and G. Garratty, "Serologic findings in autoimmune hemolytic anemia associated with immunoglobulin M warm autoantibodies," Transfusion, vol. 49, no. 2, pp. 235242, 2009.

[20] S. Zeerleder, "Autoimmune haemolytic anaemia-a practical guide to cope with a diagnosticand therapeutic challenge," Netherlands Journal of Medicine, vol. 69, no. 4, pp. 177-184, 2011.

[21] U. J. H. Sachs, L. Röder, S. Santoso, and G. Bein, "Does a negative direct antiglobulin test exclude warm autoimmune haemolytic anaemia? A prospective study of 504 cases," British Journal of Haematology, vol. 132, no. 5, pp. 655-656, 2006.

[22] F. Gilsanz, J. de La Serna, L. Moltó, and M. Alvarez-Mon, "Hemolytic anemia in chronic large granular lymphocytic leukemia of natural killer cells: cytotoxicity of natural killer cells against autologous red cells is associated with hemolysis," Transfusion, vol. 36, no. 5, pp. 463-466, 1996.

[23] H. L. Griffiths, B. M. Kumpel, C. J. Elson, and A. G. Hadley, “The functional activity of human monocytes passively sensitized with monoclonal anti-D suggests a novel role for Fc $\gamma$ RI in the immune destruction of blood cells," Immunology, vol. 83, no. 3, pp. 370-377, 1994.

[24] M. Lai, G. Leone, and R. Landolfi, "Autoimmune hemolytic anemia with gel-based immunohematology tests," American Journal of Clinical Pathology, vol. 139, no. 4, pp. 457-463, 2013.

[25] J. H. Jandl, A. R. Jones, and W. B. Castle, "The destruction of red cells by antibodies in man. I. Observations of the sequestration and lysis of red cells altered by immune mechanisms," The Journal of Clinical Investigation, vol. 36, no. 10, pp. 1428-1459, 1957.

[26] J. H. Jandl and M. E. Kaplan, "The destruction of red cells by antibodies in man. III. Quantitative factors influencing the patterns of hemolysis in vivo," The Journal of Clinical Investigation, vol. 39, pp. 1145-1156, 1960.

[27] R. J. Kurlander and W. F. Rosse, "Monocyte-mediated destruction in the presence of serum of red cells coated with antibody," Blood, vol. 54, no. 5, pp. 1131-1139, 1979.
[28] A. F. Lobuglio, R. S. Cotran, and J. H. Jandl, "Red cells coated with immunoglobulin G: binding and sphering by mononuclear cells in man," Science, vol. 158, no. 3808, pp. 1582-1585, 1967.

[29] N. Abramson, A. F. Lo Buglio, J. H. Jandl, and R. S. Cotran, “The interaction between human monocytes and red cells. Binding characteristics," The Journal of Experimental Medicine, vol. 132, no. 6, pp. 1191-1206, 1970.

[30] E. M. Meulenbroek, D. Wouters, and S. Zeerleder, "Methods for quantitative detection of antibody-induced complement activation on red blood cells," Journal of Visualized Experiments, no. 83, Article ID e51161, 2014.

[31] R. J. Kurlander, W. F. Rosse, and G. L. Logue, "Quantitative influence of antibody and complement coating of red cells on monocyte-mediated cell lysis," The Journal of Clinical Investigation, vol. 61, no. 5, pp. 1309-1319, 1978.

[32] A. D. Schreiber and M. M. Frank, "Role of antibody and complement in the immune clearance and destruction of erythrocytes. I. In vivo effects of IgG and IgM complementfixing sites," The Journal of Clinical Investigation, vol. 51, no. 3, pp. 575-582, 1972.

[33] N. Abramson, E. W. Gelfand, J. H. Jandl, and F. S. Rosen, "The interaction between human monocytes and red cells. Specificity for IgG subclasses and IgG fragments," The Journal of Experimental Medicine, vol. 132, no. 6, pp. 1207-1215, 1970.

[34] Z. Li, Z. Shao, Y. Xu et al., "Subclasses of warm autoantibody IgG in patients with autoimmune hemolytic anemia and their clinical implications," Chinese Medical Journal, vol. 112, no. 9, pp. 805-808, 1999.

[35] B. Bardill, C. Mengis, M. Tschopp, and W. A. Wuillemin, "Severe IgA-mediated auto-immune haemolytic anaemia in a 48-yr-old woman," European Journal of Haematology, vol. 70, no. 1, pp. 60-63, 2003.

[36] H. Birgens, H. Frederiksen, H. C. Hasselbalch et al., "A phase III randomized trial comparing glucocorticoid monotherapy versus glucocorticoid and rituximab in patients with autoimmune haemolytic anaemia," British Journal of Haematology, vol. 163, no. 3, pp. 393-399, 2013.

[37] A. Zanella and W. Barcellini, "Treatment of autoimmune hemolytic anemias," Hematology Education: The Education Program for the Annual Congress of the European Hematology Association, vol. 8, pp. 339-348, 2014.

[38] S. Berentsen, "Rituximab for the treatment of autoimmune cytopenias," Haematologica, vol. 92, no. 12, pp. 1589-1596, 2007.

[39] L. D. Petz and G. Garratty, "Management of autoimmune hemolytic anemias," in Immune Hemolytic Anemias, L. D. Petz and G. Garratty, Eds., pp. 401-458, Churchill Livingstone, Philadelphia, Pa, USA, 2004.

[40] G. K. Melve, T. Hervig, R. Øvrebøe, and I. Nesthus, "Blood transfusion and pretransfusion testing in patients with warm autoimmune haemolytic anaemia," Tidsskrift for den Norske Laegeforening, vol. 124, no. 22, pp. 2918-2920, 2004 (Norwegian).

[41] R. S. Shirey, J. S. Boyd, A. V. Parwani, W. S. Tanz, P. M. Ness, and K. E. King, "Prophylactic antigen-matched donor blood for patients with warm autoantibodies: an algorithm for transfusion management.," Transfusion, vol. 42, no. 11, pp. 14351441, 2002.

[42] G. Meny, "Review: transfusing incompatible RBCs: clinical aspects," Immunohematology, vol. 20, no. 3, pp. 161-166, 2004.

[43] L. D. Petz, "A physician's guide to transfusion in autoimmune haemolytic anaemia," British Journal of Haematology, vol. 124, no. 6, pp. 712-716, 2004. 
[44] U. Randen, G. Troen, A. Tierens et al., "Primary cold agglutininassociated lymphoproliferative disease: a B-cell lymphoma of the bone marrow distinct from lymphoplasmacytic lymphoma," Haematologica, vol. 99, no. 3, pp. 497-504, 2014.

[45] S. Berentsen, E. Ulvestad, R. Langholm et al., "Primary chronic cold agglutinin disease: a population based clinical study of 86 patients," Haematologica, vol. 91, no. 4, pp. 460-466, 2006.

[46] W. F. Rosse and J. P. Adams, "The variability of hemolysis in the cold agglutinin syndrome," Blood, vol. 56, no. 3, pp. 409-416, 1980.

[47] M. Harboe and J. Deverill, "Immunochemical properties of cold haemagglutinins," Scandinavian Journal of Haematology, vol. 61, pp. 223-237, 1964.

[48] C. T. Hughey, J. W. Brewer, A. D. Colosia, W. F. Rosse, and R. B. Corley, "Production of IgM hexamers by normal and autoimmune B cells: implications for the physiologic role of hexameric IgM," Journal of Immunology, vol. 161, no. 8, pp. 40914097, 1998.

[49] M. J. Stone, Y. G. McElroy, A. Pestronk, J. L. Reynolds, J. T. Newman, and A. W. Tong, "Human monoclonal macroglobulins with antibody activity," Seminars in Oncology, vol. 30, no. 2, pp. 318-324, 2003.

[50] M. Harboe, R. van Furth, H. Schubothe, K. Lind, and R. S. Evans, "Exclusive occurrence of $\mathrm{K}$ chains in isolated cold haemagglutinins," Scandinavian Journal of Haematology, vol. 2, no. 3, pp. 259-266, 1965.

[51] P. L. Swiecicki, L. T. Hegerova, and M. A. Gertz, "Cold agglutinin disease," Blood, vol. 122, no. 7, pp. 1114-1121, 2013.

[52] S. P. Treon, L. Xu, G. Yang et al., "MYD88 L265P somatic mutation in Waldenström's macroglobulinemia," The New England Journal of Medicine, vol. 367, no. 9, pp. 826-833, 2012.

[53] A. S. Wiener, L. J. Unger, L. Cohen, and J. Feldman, "Typespecific cold auto-antibodies as a cause of acquired hemolytic anemia and hemolytic transfusion reactions: biologic test with bovine red cells," Annals of internal medicine, vol. 44, no. 2, pp. 221-240, 1956.

[54] P. D. Issitt, "I blood group system and its relationship to disease," The Journal of Medical Laboratory Technology, vol. 25, no. 1, pp. $1-6,1968$.

[55] E. Ulvestad, S. Berentsen, K. Bø, and F. V. Shammas, "Clinical immunology of chronic cold agglutinin disease," European Journal of Haematology, vol. 63, no. 4, pp. 259-266, 1999.

[56] J. Jonsen, E. Kass, and M. Harboe, "Complement and complement components in acquired hemolytic anemia with high titer cold antibodies," Acta Medica Scandinavica, vol. 170, pp. 725$729,1961$.

[57] C. J. Jaffe, J. P. Atkinson, and M. M. Frank, "The role of complement in the clearance of cold agglutinin sensitized erythrocytes in man," Journal of Clinical Investigation, vol. 58, no. 4, pp. 942-949, 1976.

[58] E. Ulvestad, S. Berentsen, and T. E. Mollnes, "Acute phase haemolysis in chronic cold agglutinin disease," Scandinavian Journal of Immunology, vol. 54, no. 1-2, pp. 239-242, 2001.

[59] A. Röth, A. Hüttmann, R. P. Rother, U. Dührsen, and T. Philipp, "Long-term efficacy of the complement inhibitor eculizumab in cold agglutinin disease," Blood, vol. 113, no. 16, pp. 3885-3886, 2009.

[60] E. Ulvestad, "Paradoxical haemolysis in a patient with cold agglutinin disease," European Journal of Haematology, vol. 60, no. 2, pp. 93-100, 1998.
[61] J. Dacie, "Auto-immune haemolytic anaemia (AIHA): treatment," in The Haemolytic Anaemias, J. Dacie, Ed., vol. 3, pp. 452520, Churchill Livingstone, London, UK, 1992.

[62] S. Berentsen, E. Ulvestad, and G. E. Tjønnfjord, "B-lymphocytes as targets for therapy in chronic cold agglutinin disease," Cardiovascular \& Hematological Disorders: Drug Targets, vol. 7, no. 3, pp. 219-227, 2007.

[63] S. Berentsen, E. Ulvestad, B. T. Gjertsen et al., "Rituximab for primary chronic cold agglutinin disease: a prospective study of 37 courses of therapy in 27 patients," Blood, vol. 103, no. 8, pp. 2925-2928, 2004.

[64] C. Schollkopf, L. Kjeldsen, O. W. Bjerrum et al., "Rituximab in chronic cold agglutinin disease: a prospective study of 20 patients," Leukemia and Lymphoma, vol. 47, no. 2, pp. 253-260, 2006.

[65] M. Ghielmini, S.-F. H. Schmitz, S. B. Cogliatti et al., "Prolonged treatment with rituximab in patients with follicular lymphoma significantly increases event-free survival and response duration compared with the standard weekly $\times 4$ schedule," Blood, vol. 103, no. 12, pp. 4416-4423, 2004.

[66] N. Cooper and D. M. Arnold, "The effect of rituximab on humoral and cell mediated immunity and infection in the treatment of autoimmune diseases," British Journal of Haematology, vol. 149, no. 1, pp. 3-13, 2010.

[67] S. Berentsen, U. Randen, A. M. Vågan et al., "High response rate and durable remissions following fludarabine and rituximab combination therapy for chronic cold agglutinin disease," Blood, vol. 116, no. 17, pp. 3180-3184, 2010.

[68] K. R. Carson, L. G. Beckwith, and J. Mehta, "Successful treatment of IgM-mediated autoimmune hemolytic anemia with bortezomib," Blood, vol. 115, no. 4, p. 915, 2010.

[69] A. Gueli, D. Gottardi, H. Hu, I. Ricca, A. de Crescenzo, and C. Tarella, "Efficacy of rituximab-bendamustine in cold agglutinin haemolytic anaemia refractory to previous chemoimmunotherapy: a case report," Blood Transfusion, vol. 11, no. 2, pp. 311-314, 2013.

[70] S. Berentsen, "How I manage cold agglutinin disease," British Journal of Haematology, vol. 153, no. 3, pp. 309-317, 2011.

[71] D. Crisp and W. Pruzanski, "B-cell neoplasms with homogeneous cold-reacting antibodies (cold agglutinins)," The American Journal of Medicine, vol. 72, no. 6, pp. 915-922, 1982.

[72] F. NíÁinle, O.-P. R. Hamnvik, C. Gulmann et al., "Diffuse large B-cell lymphoma with isolated bone marrow involvement presenting with secondary cold agglutinin disease," International Journal of Laboratory Hematology, vol. 30, no. 5, pp. 444-445, 2008.

[73] A. E. Eskazan, H. Akmurad, S. Ongoren, O. Ozer, and B. Ferhanoglu, "Primary gastrointestinal diffuse large B cell lymphoma presenting with cold agglutinin disease," Case Reports in Gastroenterology, vol. 5, no. 2, pp. 262-266, 2011.

[74] J. Dacie, "Haemolytic anaemias associated with malignant lymphomas other than Hodgkin's disease and chronic lymphocytic leukaemia (CLL)," in The Haemolytic Anaemias, J. Dacie, Ed., vol. 4, pp. 27-40, Churchill Livingstone, London, UK, 1995.

[75] D. H. Linz, S. W. Tolle, and D. L. Elliot, "Mycoplasma pneumoniae pneumonia. Experience at a referral center," Western Journal of Medicine, vol. 140, no. 6, pp. 895-900, 1984.

[76] H. S. Ginsberg, "Acute hemolytic anemia in primary atypical pneumonia associated with high titer of cold agglutinins; report of a case," The New England Journal of Medicine, vol. 234, pp. 234-826, 1946. 
[77] P. Wentworth and L. R. Bate, "Acute hemolytic anemia secondary to infectious mononucleosis.," Canadian Medical Association journal, vol. 123, no. 6, pp. 482-486, 1980.

[78] J. Donath and K. Landsteiner, "Über Paroxysmale Hämoglobinurie," Münchener medizinische Wochenschrift, vol. 51, pp. 1590-1593, 1904.

[79] M. A. Gertz, "Cold hemolytic syndrome," Hematology/The Education Program of the American Society of Hematology, pp. 19-23, 2006.

[80] A. Ziman, R. Hsi, and D. Goldfinger, "Donath-Landsteiner antibody-associated hemolytic anemia after Haemophilus influenzae infection in a child," Transfusion, vol. 44, no. 8, pp. 1127-1128, 2004.

[81] M. D’Angio, T. Ceglie, G. Giovannetti et al., "Visceral leishmaniasis presenting with paroxysmal cold haemoglobinuria," Blood Transfusion, vol. 12, supplement 1, pp. s141-s143, 2014.

[82] B. Mayer, S. Yürek, H. Kiesewetter, and A. Salama, "Mixedtype autoimmune hemolytic anemia: differential diagnosis and a critical review of reported cases," Transfusion, vol. 48, no. 10, pp. 2229-2234, 2008.

[83] T. A. Shah, C. T. Mauriello, P. S. Hair et al., "Complement inhibition significantly decreases red blood cell lysis in a rat model of acute intravascular hemolysis," Transfusion, vol. 54, no. 11, pp. 2892-2900, 2014.

[84] K. Bork, I. Steffensen, and T. Machnig, "Treatment with C1-esterase inhibitor concentrate in type I or II hereditary angioedema: a systematic literature review," Allergy and Asthma Proceedings, vol. 34, no. 4, pp. 312-327, 2013.

[85] P. Hillmen, N. S. Young, J. Schubert et al., "The complement inhibitor eculizumab in paroxysmal nocturnal hemoglobinuria," The New England Journal of Medicine, vol. 355, no. 12, pp. 1233-1243, 2006.

[86] A. M. Risitano, D. Ricklin, Y. Huang et al., "Peptide inhibitors of C3 activation as a novel strategy of complement inhibition for the treatment of paroxysmal nocturnal hemoglobinuria," Blood, vol. 123, no. 13, pp. 2094-2101, 2014.

[87] D. Wouters, F. Stephan, P. Strengers et al., "C1-esterase inhibitor concentrate rescues erythrocytes from complement-mediated destruction in autoimmune hemolytic anemia," Blood, vol. 121, no. 7, pp. 1242-1244, 2013. 


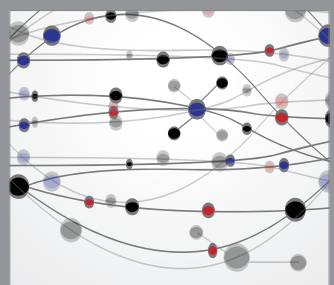

The Scientific World Journal
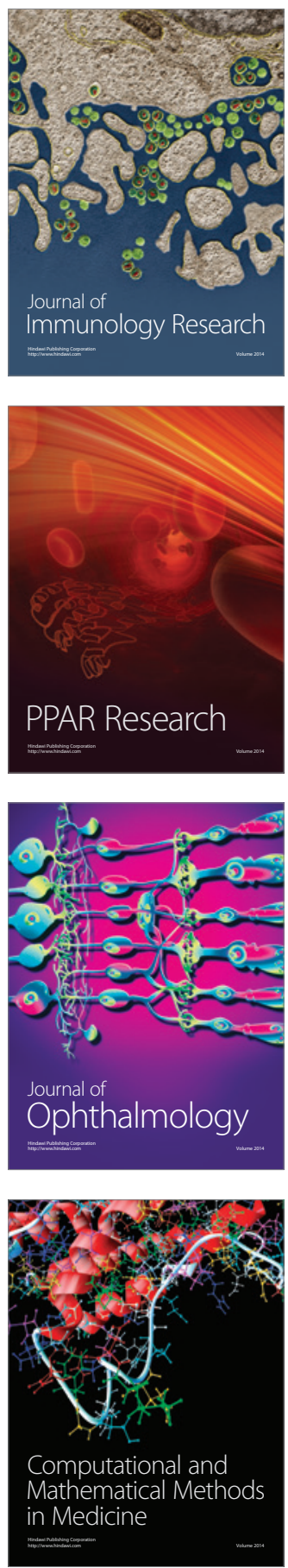

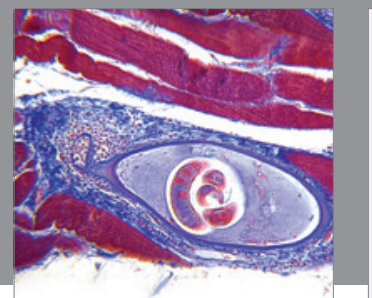

Gastroenterology

Research and Practice
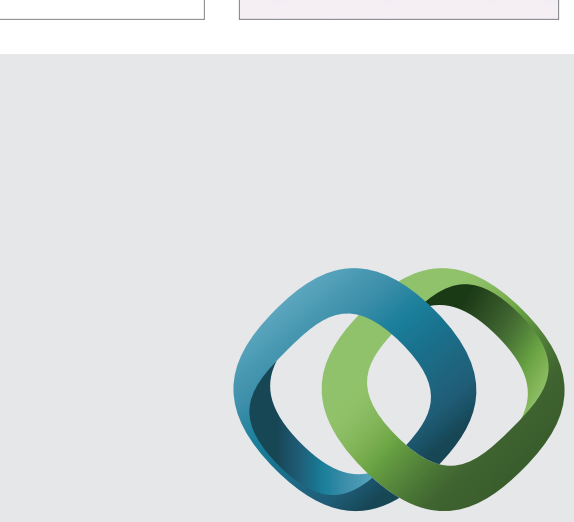

\section{Hindawi}

Submit your manuscripts at

http://www.hindawi.com
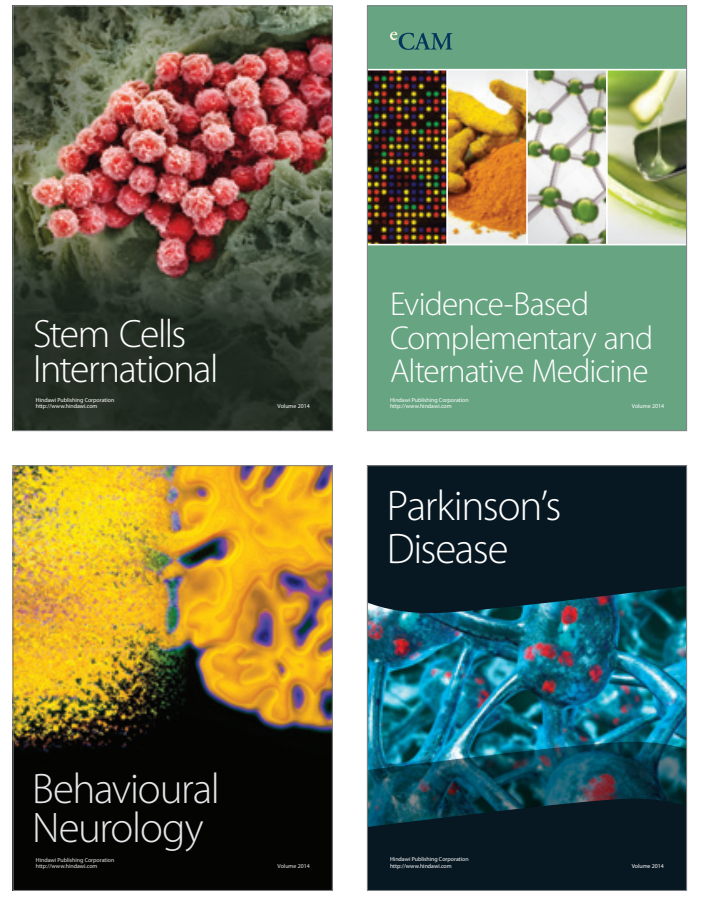
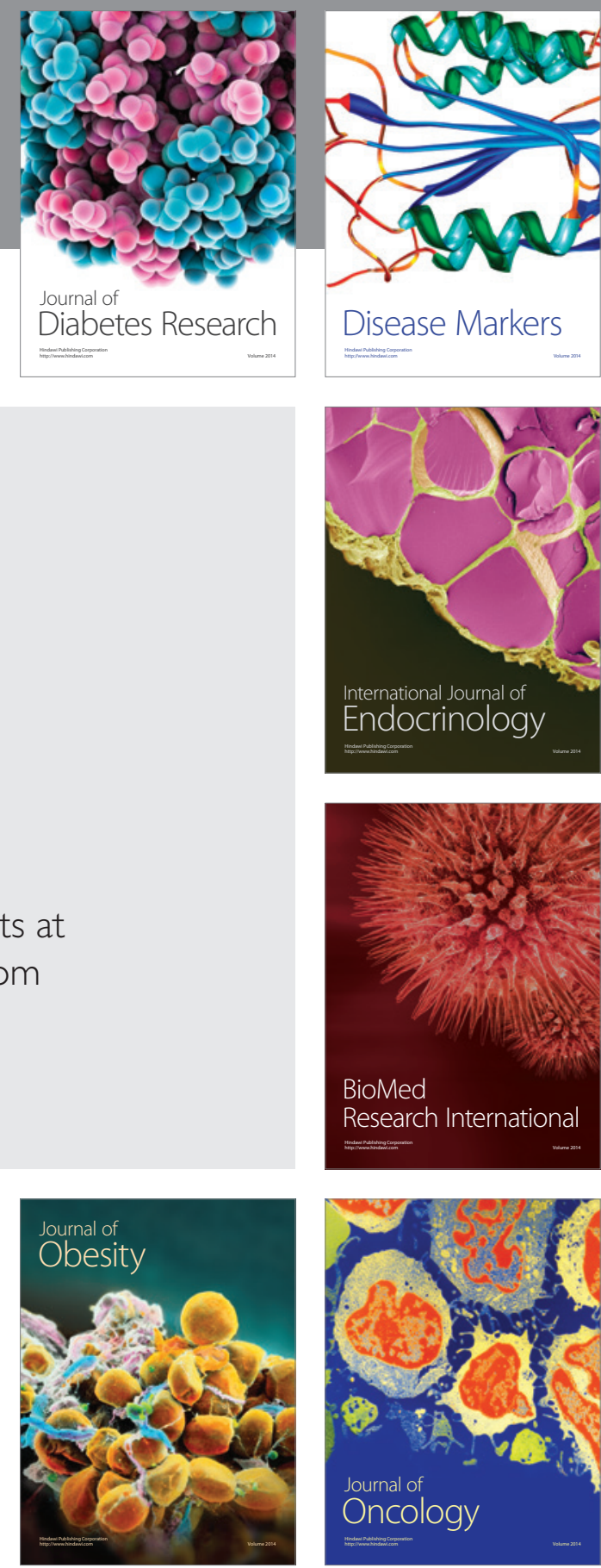

Disease Markers
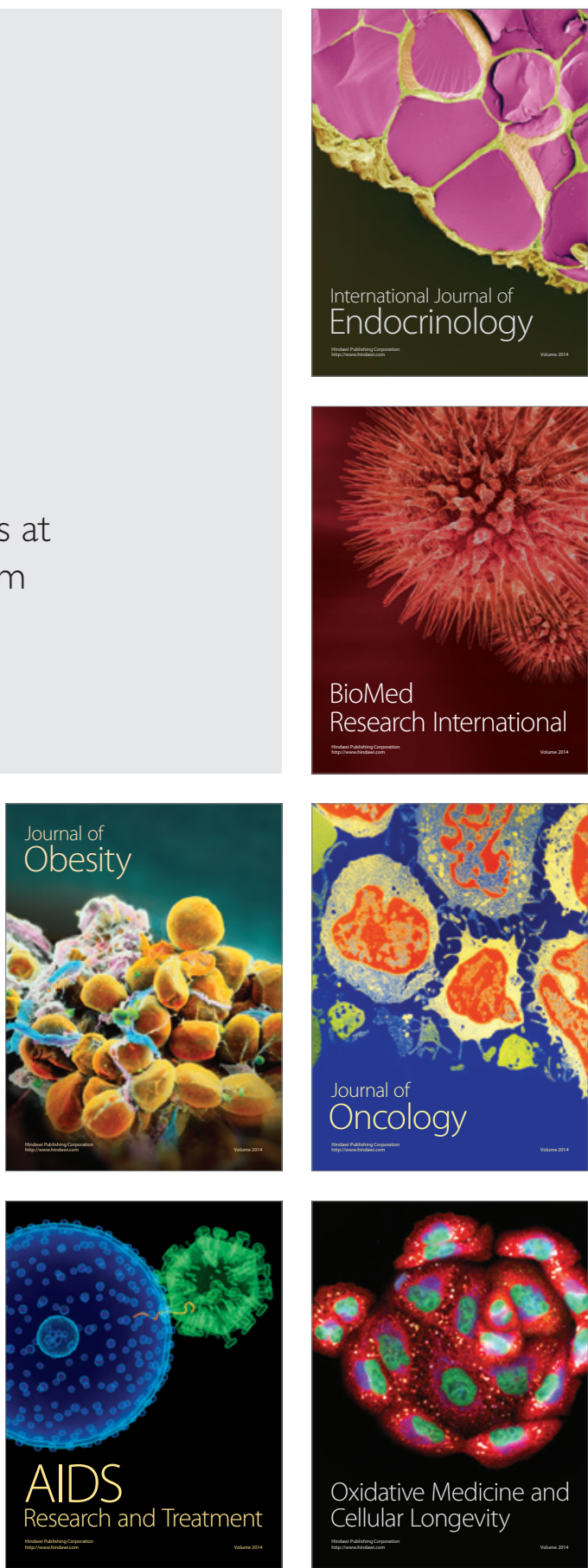